\title{
BIBLIOGRAPHIC PRODUCTION IN GEOGRAPHY OF INDUSTRY AND PRODUCTIVE SYSTEMS
}

\section{Edilson Alves Pereira Júnior e Leandro Bruno Santos}

\section{(2) OpenEdition \\ 1 Journals}

\section{Edição electrónica}

URL: http://journals.openedition.org/espacoeconomia/7855

DOI: ERREUR PDO dans /localdata/www-bin/Core/Core/Db/Db.class.php L.34 : SQLSTATE[HYO00]

[1040] Too many connections

ISSN: 2317-7837

\section{Editora}

Núcleo de Pesquisa Espaço \& Economia

\section{Refêrencia eletrónica}

Edilson Alves Pereira Júnior e Leandro Bruno Santos, «BIBLIOGRAPHIC PRODUCTION IN GEOGRAPHY OF INDUSTRY AND PRODUCTIVE SYSTEMS », Espaço e Economia [Online], 15 | 2019, posto online no dia 02 novembro 2019, consultado o 05 novembro 2019. URL : http:// journals.openedition.org/espacoeconomia/7855; DOI : 10.4000/espacoeconomia.7855

Este documento foi criado de forma automática no dia 5 novembro 2019

(C) NUPEE 


\title{
BIBLIOGRAPHIC PRODUCTION IN GEOGRAPHY OF INDUSTRY AND PRODUCTIVE SYSTEMS
}

\author{
Edilson Alves Pereira Júnior e Leandro Bruno Santos
}

\section{MERCATOR, Revista de Geografia da Universidade Federal do Ceará (UFC)}

\section{Link para a revista:}

1 http://www.mercator.ufc.br/mercator/article/view/e18014

\begin{abstract}
2 The productive processes and their relations with the geographic space underwent profound changes in the last decades of the twentieth century. The mentioned changes substantialy altered the dynamics of the space economy, making them less predictable and increasingly open to thematic and theoretical-methodological changes in the studies on Geography of Industries. Considering those transformations, this paper represents an effort to outline the recent Geography of Industries bibliographic production in Brazil, inside the context of the greater knowledge dissemination through the information technologies, the consolidation of traditional study centers and the expansion of graduate programs in Geography. The database was created through the Google Scholar Platform, including the publications of 60 graduate programs in Geography from the period between 2005 and 2016. We attempted to capture and analyze subthemes, interpretation lines, authors, published papers, quotations and research centers on Geography of Industries and productive systems. The results point out the greater diversity of the research lines and thematic clippings, the consolidation of new theoretical influences and the greater participation of many
\end{abstract}


country regions in the bibliographic production on the theme.

Keywords: $\mathrm{H}$ index, Graduate programs in Geography, Geography of Industries, Brazil.

\section{BIBLIOGRAFIA}

ARAÚJO, Tânia Bacelar. Ensaios sobre o desenvolvimento brasileiro: heranças e urgências. Rio de Janeiro: Revan/Fase, 2000. 390p.

ARROYO, María Mónica. A globalização pensada a partir do espaço geográfico. In: MENDONÇA, Francisco de Assis et al (Org.). Espaço e tempo: complexidade e desafios do pensar e do fazer geográfico. Curitiba: Ademadan, 2009. p. 479-496.

AZZONI, Carlos R. Teoria da localização: uma análise crítica. A experiência de empresas instaladas no Estado de São Paulo. São Paulo: Instituto de Pesquisas Econômicas, 1982. 200p.

BARNES, Trevor John. Economic geography. In: KITCHIN, Rob; THRIFT, Nigel (Org.). International encyclopedia of human geography. London: Elsevier, 2009. p. 315-327.

BECKER, Bertha K. Crescimento econômico e estrutura espacial do Brasil. Revista Brasileira de Geografia, v. 34, n. 4, p. 101-116, 1972.

BECKER, Bertha K.; BERNARDES, Nilo. Considerações sobre o desenvolvimento regional e a localização espacial das atividades nos países em desenvolvimento. Revista Brasileira de Geografia, v. 41, n. 3, p. 135-150, 1976.

BENKO, Georges. Economia, espaço e globalização na aurora do século XXI. São Paulo: Hucitec, 1996. 266p.

BENKO, Georges. La géographie économique: un siècle d'histoire. Annales de Géographie, n. 664, p. 23-49, 2008.

BENKO, Georges. SCOTT, Allen J. La géographie économique: traditions et turbulences. In: BENKO, Georges; STROHMAYER. Ulf. Horizons géographiques. Paris: Bréal, 2004. p. 151-191.

CAMPOS, Maria da Glória de Carvalho. Causas geográficas do desenvolvimento das olarias na baixada da guanabara. Revista Brasileira de Geografia, v. 17, n. 2, p. 123- 151, 1955.

CAPEL, Horacio. Geografía y economía. In: CAPEL, Horacio. Geografía humana y ciencias sociales. Una perspectiva histórica. Barcelona: Montesinos, 1984, p. 83-122.

CARAVACA, Inmaculada. Los nuevos espacios ganadores y emergentes. Eure, v. 24, n. 73, p. 5-30, 1998.

CASTELLS, Manuel. A sociedade em rede - a era da informação. São Paulo: Paz \& Terra, 1999. 698p.

CHANG, Stephen S. O papel dos geógrafos "culturais" nas decisões industriais. Revista Brasileira de Geografia, v. 40, n. 1, p. 155-161, 1978.

CLAVAL, Paul. Geografia econômica e economia. Geotextos, v. 1, n. 1, p. 11-27, 2005.

CLAVAL, Paul. A diversidade das geografias econômicas. Geographia, v. 14, n. 2, p. 7-20, 2012.

CORRÊA, Roberto Lobato. O enfoque locacional na geografia. Terra Livre, n.1, p. 62-66, 1986.

DAVIDOVICH, Fany. Aspectos geográficos de um centro industrial: Jundiaí em 1962. Revista Brasileira de Geografia, v. 28, n. 4, p. 329-374, 1966,

DINIZ, Clélio Campolina. A nova geografia econômica do Brasil. In: VELLOSO, João Paulo dos Reis (Org.). Brasil 500 anos: futuro, presente, passado. Rio de Janeiro: José Olympio, 2000. p. 303-351. FAISSOL, Speridião. Pólos de desenvolvimento no Brasil: uma metodologia quantitativa e uma 
exemplificação empírica. Revista Brasileira de Geografia, v. 34, n. 2, p. 52-80, 1972.

GEIGER, Pedro P. Estudos para a geografia das indústrias do Brasil Sudeste. Revista Brasileira de Geografia, v. 25, n. 2, p. 155-276, 1963.

GEIGER, Pedro Pinchas. Contribuição aos estudos da estrutura espacial do sistema industrial no Brasil (A criação de um sistema de dados). Revista Brasileira de Geografia, v. 44, n.2, p. 317-329, 1982.

GEIGER, Pedro Pinchas. Industrialização, urbanização e a persistência das desigualdades regionais do Brasil. Revista Brasileira de Geografia, v. 38, n. 2, p. 3-99, 1976.

GEIGER, Pedro Pinchas. Questões geográficas da concentração dos estabelecimentos industriais. Revista Brasileira de Geografia, v. 42, n.2, p. 310-331, 1980.

GEIGER, Pedro Pinchas. Urbanização e industrialização na Orla Oriental da Baía de Guanabara. Revista Brasileira de Geografia, v. 18, n. 4, p. 495-522, 1956.

GEIGER, Pedro Pinchas; DAVIDOVICH, Fany. Reflexões sobre a evolução da estrutura espacial do Brasil sob o efeito da industrialização. Revista Brasileira de Geografia, v. 36, n.3, p. 3-29, 1974. HADDAD, Paulo Roberto. Padrões regionais de crescimento do emprego industrial de 1950 a 1970. Revista Brasileira de Geografia, v. 39, n. 1, p. 3-45, 1977.

JATOBA, Jorge. Emprego e industrialização: a experiência da região metropolitana do Recife (RMR) 1950-1970. Revista Brasileira de Geografia, v. 39, n. 4, p. 181-214, 1977.

LENCIONI, Sandra. Cisão territorial da indústria e integração regional no Estado de São Paulo. In: GONÇALVES, Maria Flora et al. (Org.). Regiões e cidades, cidades nas regiões: o desafio urbanoregional. São Paulo: UNESP/ANPUR, 2003. p. 465-475.

LENCIONI, Sandra. Novos rumos e tendências da urbanização e a industrialização no Estado de São Paulo. In: LIMONAD, Ester; HAESBAERT, Rogério; MOREIRA, Ruy (Org.). Brasil século XXI: por uma nova regionalização? agentes, processos e escalas. São Paulo: Max Limonad, 2004. p. 67-77. LENCIONI, Sandra. Reestruturação urbano-industrial no estado de São Paulo: a região da metrópole desconcentrada. Espaço e Debates, n.38, p.54-61, 1994.

LIMA, Luiz Cruz. Novo espaço da produção: os tecnopolos. São Paulo: Universidade de São Paulo, 1994. Tese (Doutorado em Geografia) - FFLCH/USP, São Paulo, 1994

MAGALHÃES, J. CEZAR DE. A função industrial de Petrópolis. Revista Brasileira de Geografia, v. 28, n. 1, p. 19-55, 1966.

MAGALHÃES, José Cezar de. Energia elétrica: fator de desenvolvimento industrial na zona metalúrgica de Minas Gerais. Revista Brasileira de Geografia, v. 31, n. 1, p. 26-42, 1969.

MAIMON, Dalia. Considerações metodológicas sobre tamanho de firma. Revista Brasileira de Geografia, 1977, v. 39, n. 4, p. 151-163.

MAMIGONIAN, Armen. Estudo geográfico das indústrias de Blumenau. Revista Brasileira de Geografia, v. 27, n. 3, p. 389-481, 1965.

MANZAGOL, Claude. Lógica do espaço industrial. São Paulo: Difel, 1985. 230p.

MARTIN, Ron. Teoria econômica e geografia humana. In: GREGORY, Derek; MARTIN, Ron. SMITH, Graham (Org.). Geografia humana: sociedade, espaço e ciência social. Rio de Janeiro: Jorge Zahar Editor, 1996. p. 31-64.

MELO, Mário Lacerda de. Aspectos da geografia do açúcar no Brasil. Revista Brasileira de Geografia, v. 16, n. 4, p. 467- 492, 1954.

MÉNDEZ, Ricardo e CARAVACA, Inmaculada. Organización industrial y território. Madrid: Editorial Síntesis, 1996. 365p.

MÉNDEZ, Ricardo. Geografía económica - la lógica espacial del capitalismo global. Barcelona: Editora Ariel, 1997. 384p.

MOLD, Zilá Mesquita. Para um novo enfoque de diversificação e especialização industrial. Revista Brasileira de Geografia, v. 39, n. 3, p. 151-174, 1977.

OLIVEIRA, Lucia Elena Garcia de. Algumas considerações sobre a implantação de distritos industriais. Revista Brasileira de Geografia, v. 38, n. 4, p. 22-69, 1976. 
PORCARO, Rosa Maria. Industrialização e tamanho urbano. Revista Brasileira de Geografia, v. 39, n. 1, p. 46-86, 1977.

RAMIRES, Julio Cesar Lima. As corporações multinacionais e a organização espacial: uma introdução. Revista Brasileira de Geografia, v. 51, n.1, p. 103-112, 1989.

RIBEIRO, Miguel Ângelo Campos. Padrões de localização e estrutura de fluxos dos estabelecimentos industriais na Região Metropolitana de Salvador. Revista Brasileira de Geografia, v. 44, n.4, p. 591-637, 1982.

RIBEIRO, Miguel Angelo Campos; ALMEIDA, Roberto Schmidt de. Padrões de localização espacial e estrutura de fluxos dos estabelecimentos industriais da Área Metropolitana de Recife. Revista Brasileira de Geografia, v. 42, n.2, p. 203-264, 1980.

ROCHA, Roberto Vasconcelos Moreira da. Padrões de localização industrial e o planejamento regional. Revista Brasileira de Geografia, v. 37, n. 1, p. 123-191, 1975.

ROCHA, Sônia. Evolução das indústrias de transformação de Pernambuco entre 1970 e 1974.

Revista Brasileira de Geografia, v. 42, n. 1, p. 52-78, 1980.

ROWTHORN, Robert. Indústria de transformação: crescimento, comércio e mudança estrutural. In: Castro et al (Ed.). O Futuro da indústria no Brasil e no mundo - os desafios do século XXI. Rio de Janeiro: CNI e Campus, 1999. p. 57-76.

SAMPAIO, Silva Selingardi. Considerações em torno da Geografia industrial: teoria, métodos e uma nova técnica de mensuração da atividade fabril. Revista Brasileira de Geografia, v. 37, n. 1, p. 95-122, 1975.

SAMPAIO, Silvia Selingardi. Indústria e território em São Paulo: A estruturação do multicomplexo territorial industrial paulista (1950-2005). Campinas: Editora Alíneas, 2009. 482p.

SANTOS, Milton. Localização Industrial em Salvador. Revista Brasileira de Geografia, v. 20, n. 3, p. 245-276, 1958.

SANTOS, Milton; SILVEIRA, Maria Laura. Brasil: território e sociedade no início do século XXI. Rio de Janeiro: Record, 2001. 473p.

SCOTT, Allen. Economic geography: The great half-century. In: CLARK, Gordon L.; FEDMAN, Maryann P.; GERTLER, Meric S. (Org.). The Oxford Handbook of Economic Geography. Oxford: Oxford University Press, 2003. p. 18-44.

SPOSITO, Eliseu Savério. Território, logística e mundialização. In: SPOSITO, Eliseu Savério (Org.). Dinâmica Econômica, poder e novas territorialidades. Presidente Prudente: GasPERR/Unesp, 1999. p. 99 -113.

SPOSITO. Eliseu Savério. Fluxos e localização industrial. In: MELO, Jayro Gonçalves (Org.). Região, cidade e poder. Presidente Prudente: GASPERR, 1996. p. 69-96.

STEFFAN, Élvia Roque. Produção industrial e número de estabelecimentos em Goiás. Revista Brasileira de Geografia, v. 29, n. 2, p. 84-87, 1967.

TOLOSA, Hamilton C. Diferenciais de produtividade industrial e estrutura urbana. Revista Brasileira de Geografia, v. 36, n. 2, p. 37-56, 1974.

\section{AUTORES}

\section{EDILSON ALVES PEREIRA JÚNIOR}

Universidade Estadual do Ceará, Fortaleza (CE), Brasil

http://orcid.org/0000-0003-4734-5500

\section{LEANDRO BRUNO SANTOS}

Universidade Federal Fluminense, Campos Goytacazes (RJ), Brasil

http://orcid.org/0000-0001-9163-8568 